\title{
Novel interactive eye tracking game for training attention in children with ADHD
}

\author{
Alba García ${ }^{1,2}$, Tomas D’Amelio ${ }^{3}$, Isadora Oliveira ${ }^{4}$, Peter Collins $^{5}$, Elizabeth Liddle ${ }^{5}$, \\ Hans Supèr2,6-8 1) University Pompeu Fabra, Barcelona, Spain; 2) Braingaze S.L., Mataró, Spain; 3) Cátedra de Neurofisiología, Facultad de Psicología, Universidad de Buenos \\ Aires, Ciudad Autónoma de Buenos Aires, Argentina, 4) Department of Biomedical Engineering, School of Engineering, Trinity College Dublin, Ireland; 5) Division of \\ Psychiatry and Applied Psychology, School of Medicine, University of Nottingham, Nottingham, UK; 6) ICREA, Barcelona, Spain; 7) Instituto de Neurociencias, \\ Barcelona, Spain; 8) Department of Cognition, Development and Educational Psychology, Faculty of Psychology, University of Barcelona, Barcelona, Spain
}

\begin{abstract}
Attention Deficit Hyperactivity Disorder (ADHD) is one of the most common neurodevelopmental disorders. Existing treatments for ADHD focus on pharmaceutical medication and cognitive behavioral treatment. Pharmaceutical treatment is in many cases effective at reducing ADHD symptoms, however many families prefer not to choose it because of its chemical nature.
\end{abstract}

The usage of serious games as an alternative treatment has received considerable interest. RECOGNeyes is a computer game that is played using your eyes as the game controller. The rationale behind the training game is that individuals with ADHD have an underdeveloped attention control system. This attention control system is underdeveloped not because they lack this capacity but because this ability has not been sufficiently developed. RECOGNeyes isolates the underdeveloped attention control system and provides an opportunity to train it. The game has been developed as an intervention for training visual attention in ADHD.

Objective: To assess the therapeutic benefits of RECOGNeyes for ADHD children.

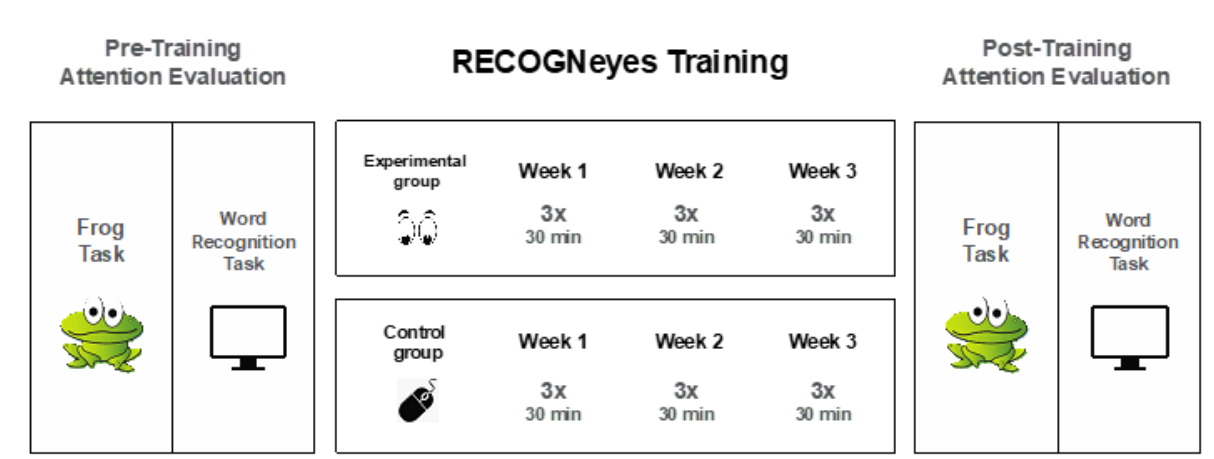

Figure 1. The study design. In the study design there are two groups (experimental and control) that were assessed with the same attentional tasks, but they played different versions (eye-tracker version or mouse version) of RECOGNeyes.

Methods: ADHD children $(\mathrm{N}=14)$ played RECOGNeyes with eye-tracker for three weeks (3 times/week for 30 minutes), while the sex/age-matched control group $(\mathrm{N}=14)$ played the game using the mouse. Two eye tracking tasks (Braingaze, Spain) were used for pre- and post-training assessment. These tasks measure a neural marker of attention as a support tool for ADHD diagnosis.

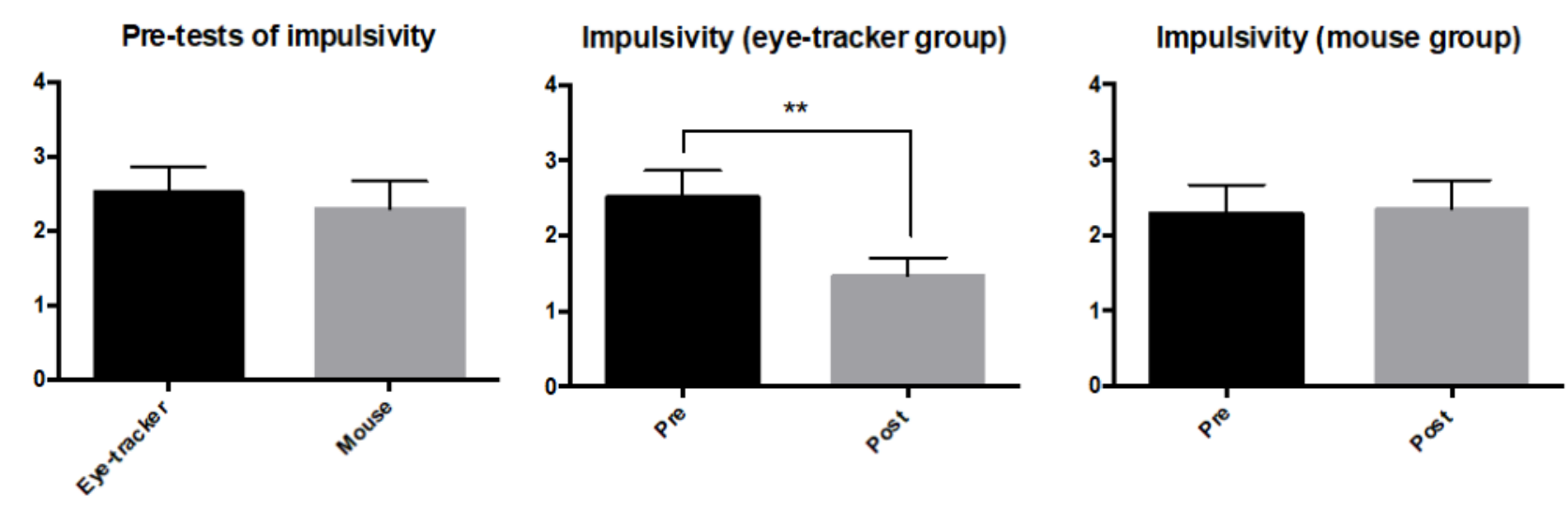

Figure 2. Impulsivity parameter. The left graph shows a comparison between eye-tracker (black bar, $\mathrm{N}-14$ ) and mouse groups (grey bar, $\mathrm{N}$ 14) pre-testing. The middle and right graphs show a comparison between pre (black bars) and post-testing (grey bars4) in eye-tracker and mouse group, respectively. Impulsivity has been significantly decreased in eye-tracker group post-testing compared to pre-testing, while no significantly changes have been shown in mouse group. ${ }^{* *}=$ $p<0.01$.

Reference: García-Baos A et al., (2019) Novel Interactive EyeTracking Game for Training Attention in Children With AttentionDeficit/Hyperactivity Disorder. Prim Care Companion CNS Disord.21(4). pii: 19m02428. doi: 10.4088/PCC.19m02428.
Results: Impulsivity showed significant differences between pre and post-test in eye-tracker group $(p=0.0067)$, but not in mouse group $(p=0.3349)$. For number of fixations, significant group and pre-and post-assessment differences were observed $(F[1,930]=$ $6.015, p=0.0144 ; \mathrm{F}[1,930]=10.23, p=0.0014$, respectively). Post-hoc analysis revealed that in both tasks the eye-tracker group (but not in the mouse group) significantly decreased the number of fixations in post-test compared to pre-test $(p<0.01)$. For duration of fixations, significant group and pre/post differences were observed $(\mathrm{F}[1,930]=45.93, p=0.0001 ; \mathrm{F}$ $[1,930]=6.554, p=0.0106$, respectively). The eye-tracker group, but not the mouse group, increased the duration of fixations in post-test compared to pre-test $(p<0.01)$. Significant group differences in reaction time were observed $(F[1,665]=11.69$, $p=0.0007)$. Post-hoc analysis showed faster reaction times $(p<0.0001)$ for the eye-tracker group in both tasks.

Conclusions: RECOGNeyes might be used as an alternative therapy to support the current treatment of ADHD.
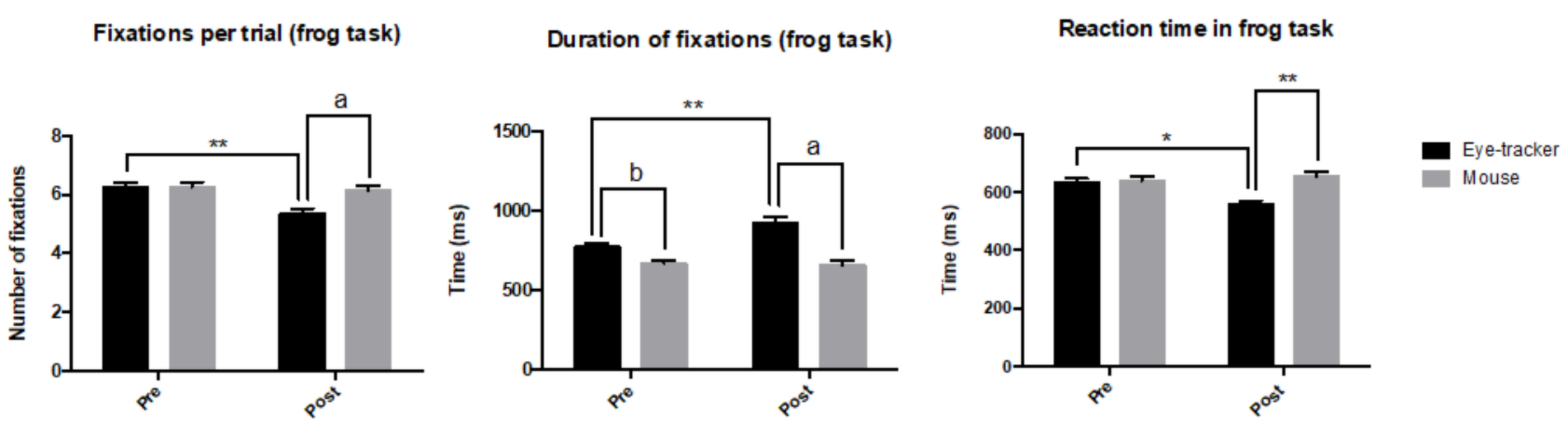

Figure 3. The number (left panel) and durations of fixations (mid panel), and reaction time (right panel) per trial in frog task for the eyetracking (black) and mouse (grey) groups at pre- and post-test time points. For the number of fixations the eye-tracking group showed a decrease between pre-and post-testing and had significantly less fixations at post-test compared to the mouse group. For duration of fixations, group differences were observed at pre- and post-tests. Only the eye-tracking group increased in post-test compared to pre-test. ${ }^{* *}=p<0.01, a=p<0.001, b=<0.05$. Participants from the eye-tracker group spent less time to respond in post- than in pre-testing, and they spent less time to respond in post-testing compared to the mouse group. ${ }^{*}=p<0.05$, differences between pre and post-test in eyetracker. $a=p<0.01$, differences between groups in post-test.

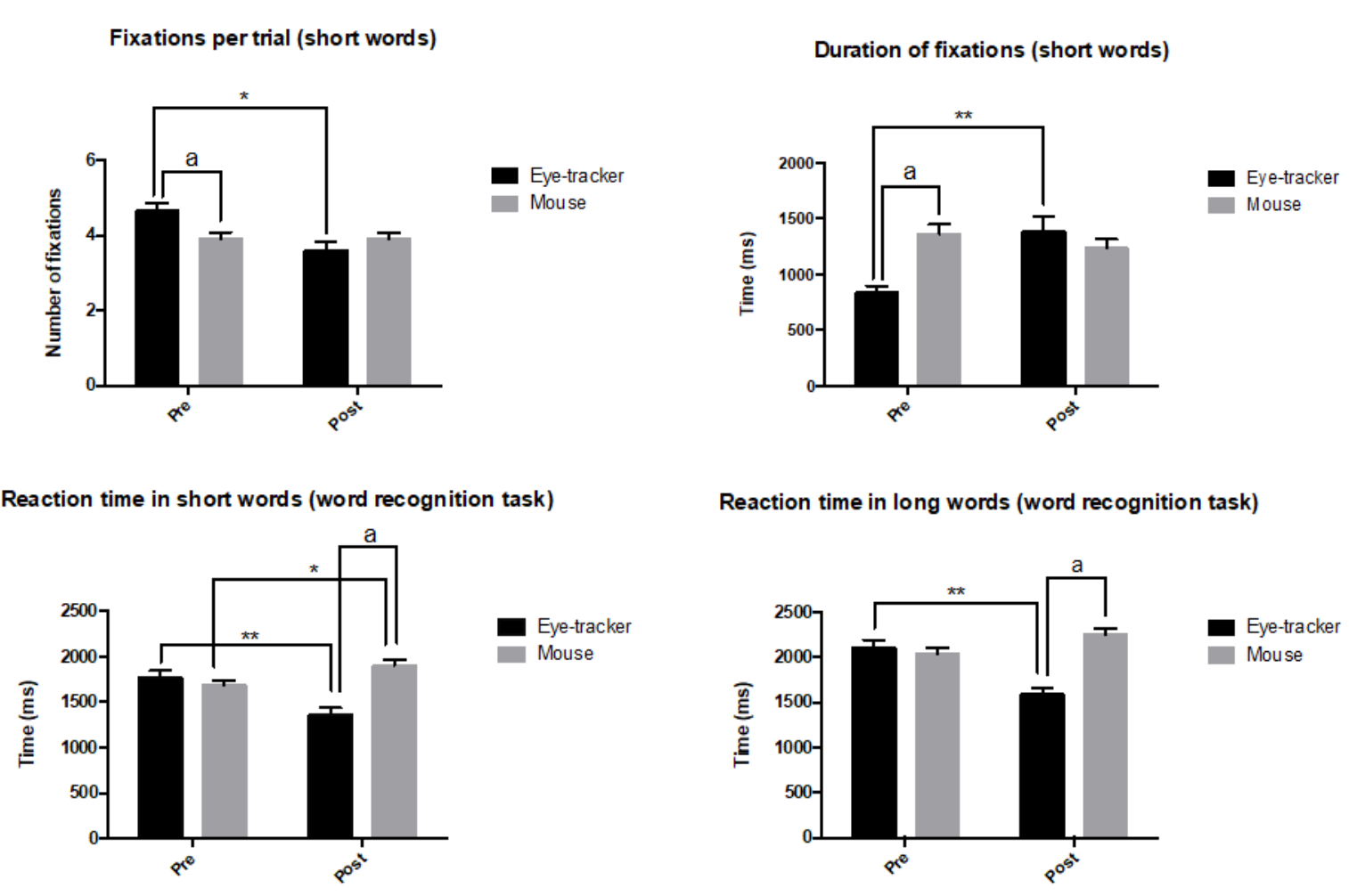

Figure 4. Number and duration of fixations on short and long words and reaction in word recognition task. Only the eye-tracker group showed a decrease in the number of fixations while an increase in its duration in post-testing compared to pre-testing. Participants from the eye-tracker group spent less time to respond in post-testing compared to pre-testing, as well as to respond in post-test compared to the mouse group. In contrast, participants from the mouse group spent more time to respond to the short words in post-testing compared to pre-testing. ${ }^{*}=p<0.05 ;{ }^{* *}=p<0.01$, differences between pre and post-test in groups. $a=p<0.001$, differences between groups in post-test. 